Table 1 A review of coexisting CHL and NLPHL cases

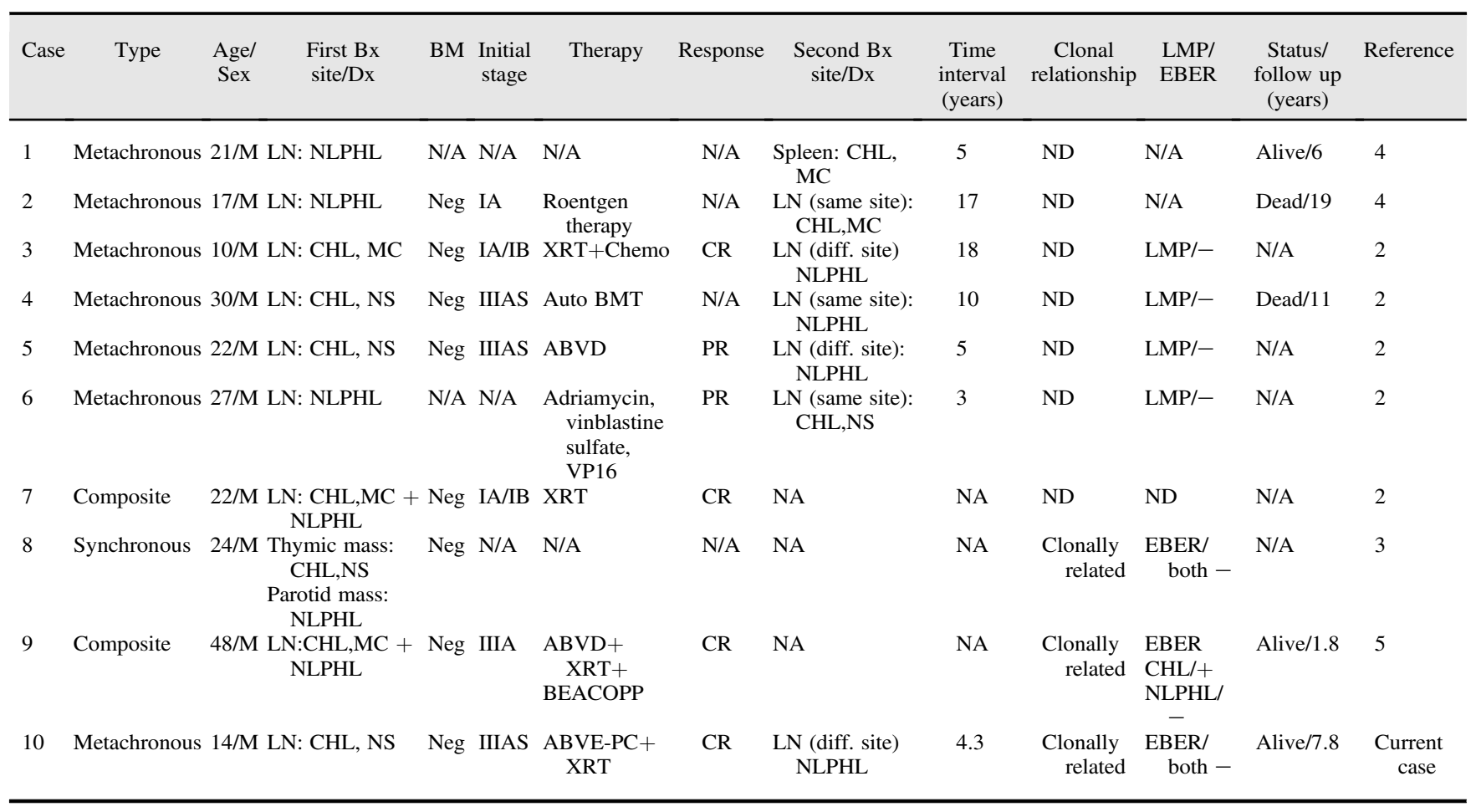

ABVD, adriamycin, bleomycin, vinblastine sulfate, dacarbazine; ABVE-PC, adriamycin, bleomycin, vincristine sulfate, etoposide, prednisone and cyclophosphamide; BEACOPP, cyclophosphamide, doxorubicin, etoposide phosphate, natulan, vincristine, bleomycin; BMT, bone marrow transplant; Bx, biopsy; Chemo, chemotherapy; CHL, classic Hodgkin lymphoma; CR, complete remission; diff, different; Dx, diagnosis; EBER, EBV-encoded RNA; F, female; LMP, latent membrane protein; LN, lymph node; M, male; MC, mixed-cellularity; NA, not applicable; N/A, not available; ND, not done; Neg, negative; NLPHL, nodular lymphocyte predominant Hodgkin lymphoma; NS, nodular sclerosis; PR, partial remission; XRT, radiotherapy.

recurrent disease, biopsy of the relapsed lesion is essential for diagnosis and treatment purposes.

Conflicts of interest and sources of funding: The authors state that there are no conflicts of interest to disclose.

\section{Yi Ding ${ }^{1}$, Andrew Campbell ${ }^{1}$, Xuan Zhang ${ }^{2}$, Kai Fu ${ }^{2}$, Ji Yuan ${ }^{2}$}

${ }^{I}$ Department of Pathology, Geisinger Health System, Danville, PA, USA; ${ }^{2}$ Department of Pathology and Microbiology, University of Nebraska Medical Center, Omaha, NE, USA

Contact Ji Yuan, MD, PhD.

E-mail: yuan.ji@mayo.edu

1. Swerdlow SH, Campo E, Harris NL, et al. WHO Classification of Tumours of Haematopoietic and Lymphoid Tissues. Revised $4^{\text {th }}$ ed. Lyon: Internation Agency for Research on Cancer, 2017; 424

2. Gelb AB, Dorfman RF, Warnke RA. Coexistence of nodular lymphocyte predominance Hodgkin's disease and Hodgkin's disease of the usual type. Am J Surg Pathol 1993; 17: 364-74

3. Song JY, Eberle FC, Xi L, et al. Coexisting and clonally identical classic hodgkin lymphoma and nodular lymphocyte predominant hodgkin lymphoma. Am J Surg Pathol 2011; 35: 767-72.

4. Miettinen M, Franssila KO, Saxen E. Hodgkin's disease, lymphocytic predominance nodular. Increased risk for subsequent non-Hodgkin's lymphomas. Cancer 1983; 51: 2293-300.

5. Szczepanowski M, Masque-Soler N, Oschlies I, et al. Composite lymphoma of nodular lymphocyte-predominant and classical Hodgkin lymphoma-Epstein-Barr virus association suggests divergent pathogenesis despite clonal relatedness. Hum Pathol 2013; 44: 1434-9.
6. Bernard SM, Cartwright RA, Darwin CM, et al. Hodgkin's disease: case control epidemiological study in Yorkshire. Br J Cancer 1987; 55: $85-90$.

7. Kerzin-Storrar L, Faed MJ, MacGillivray JB, et al. Incidence of familial Hodgkin's disease. Br J Cancer 1983; 47: 707-12.

8. Goldin LR, Pfeiffer RM, Gridley G, et al. Familial aggregation of Hodgkin lymphoma and related tumors. Cancer 2004; 100: 1902-8.

DOI: https://doi.org/10.1016/j.pathol.2020.06.019

\section{The lipid profile in children prior to isotretinoin therapy: an opportunity to detect familial hypercholesterolaemia}

Sir,

Familial hypercholesterolaemia (FH) is an autosomal dominant disorder of low density lipoprotein (LDL) catabolism associated with elevated LDL cholesterol and premature atherosclerotic coronary artery disease (CAD). ${ }^{1}$ FH has been shown to be more prevalent than previously thought, occurring in $\sim 1: 250$ people. ${ }^{2,3}$ However, the vast majority of individuals with $\mathrm{FH}$ are undiagnosed and often under treated. A recent study demonstrated that initiation of statin therapy in childhood was associated with reduced coronary artery disease. ${ }^{4} \mathrm{FH}$ is predominantly caused by variants in the LDL receptor $(L D L R)$, apolipoprotein $\mathrm{B}(A P O B)$ or proprotein 
convertase subtilisin/kexin type 9 (PCSK9) genes. ${ }^{1}$ There are multiple diagnostic criteria for $\mathrm{FH}$, with the Dutch Lipid Clinic Network Criteria preferred for Australia, although there is no international consensus. ${ }^{5}$

An international consensus guideline on optimising the detection and management of children and adolescents with FH has been published by the European Atherosclerosis Society (EAS). ${ }^{6}$ This report highlighted the need to detect and treat children and adolescents with $\mathrm{FH}$ and proposed simple LDL cholesterol based criteria to alert clinicians when an individual is at risk of $\mathrm{FH}$. A child with an LDL cholesterol $\geq 5.0 \mathrm{mmol} / \mathrm{L}$ on two successive occasions indicates probable $\mathrm{FH}$, after excluding secondary causes of hypercholesterolaemia. FH is very likely in a child with an LDL cholesterol $\geq 4.0 \mathrm{mmol} / \mathrm{L}$, if they have a parent with a history of elevated cholesterol or premature CAD. An LDL cholesterol $\geq 3.5 \mathrm{mmol} / \mathrm{L}$ in a child of a parent with genetically confirmed FH indicates probable FH. However, most adults with FH are currently undiagnosed, so identifying children may allow opportunistic reverse cascade testing of their parents, as illustrated in the following case.

A 16-year-old girl was referred to a Lipid Disorders Clinic having been found to have significant hypercholesterolaemia on routine biochemical testing prior to commencing isotretinoin therapy for acne. A fasting lipid profile showed a total cholesterol of $8.0 \mathrm{mmol} / \mathrm{L}$, HDL cholesterol $1.6 \mathrm{mmol} / \mathrm{L}$, triglyceride $1.8 \mathrm{mmol} / \mathrm{L}$, and LDL cholesterol $5.6 \mathrm{mmol} / \mathrm{L}$. The only significant past medical history was asthma and her medications included a salbutamol inhaler, fish oils $750 \mathrm{mg}$ per day, and Diane 35 (oral contraceptive pill). Her mother was known to have hypercholesterolaemia, but was not on lipid lowering therapy and her maternal grandparents were alive in their sixties. There was no family history of premature CAD. On physical examination, she appeared in good health, with a body mass index of $21 \mathrm{~kg} / \mathrm{m}^{2}$ and BP 104/61 $\mathrm{mmHg}$. There were no peripheral stigmata of lipid disorders. A cardiovascular respiratory examination was unremarkable. Based on a Dutch Lipid Clinic Network Criteria score of four, she was given a diagnosis of possible FH. Subsequent analysis of the $L D L R$ gene revealed that both she and her mother were heterozygous for a missense variant c.661G $>A$ (p.D221N) in exon 4, which occurs within repeat 5 of the ligand binding domain and which was predicted to be pathogenic.

As a result of this case, we sought to explore the potential role dermatologists could play in the opportunistic detection of children with FH using the LDL cholesterol results and clinical information on laboratory request forms for individuals aged $<18$ years from a community laboratory. The population consisted of 84,823 individuals who had plasma lipid profiles measured between 1 May 2010 and 31 April 2011 at St John of God Pathology in Western Australia. The demographics of the population have been previously described; their mean age was $56 \pm 15$ years with a mean LDL cholesterol of $3.1 \mathrm{mmol} / \mathrm{L}$.

There were 444 children aged $<18$ years in this population; their mean age was $14.9 \pm 2.4$ years, $211(47.5 \%)$ were male, with a mean total cholesterol of $4.21 \pm 0.93 \mathrm{mmol} / \mathrm{L}, \mathrm{LDL}$ cholesterol of $2.46 \pm 0.80 \mathrm{mmol} / \mathrm{L}$, and triglycerides of $1.02 \pm 0.62 \mathrm{mmol} / \mathrm{L}$. The distribution of LDL cholesterol results is shown in Fig. 1. There were $43(9.7 \%)$ children with an $\mathrm{LDL}$ cholesterol $\geq 3.5 \mathrm{mmol} / \mathrm{L}, 18(4.1 \%) \geq 4.0 \mathrm{mmol} / \mathrm{L}$, and two $(0.5 \%) \geq 5.0 \mathrm{mmol} / \mathrm{L}$.

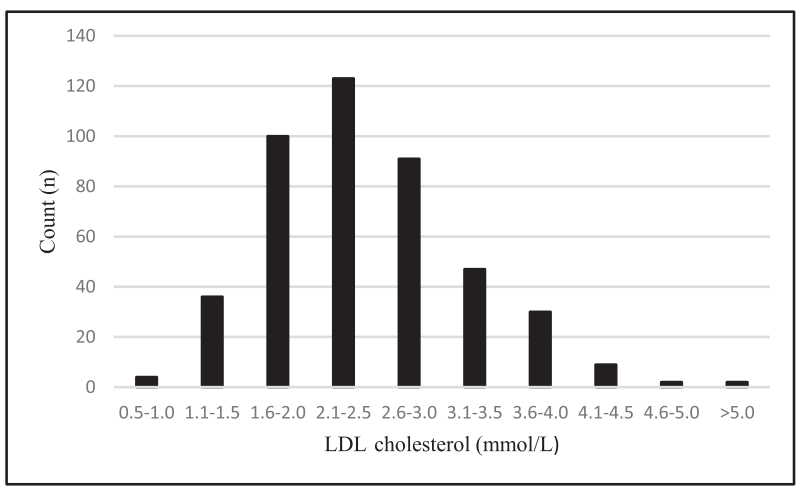

Fig. 1 Distribution of plasma LDL cholesterol concentrations in 444 children $<18$ years over a one year period in a community laboratory.

There were 96 (21.6\%) lipid profiles requested by dermatologists; 64 males and 32 females, mean age 15.5 years (range 12-17). In these 96 individuals, the mean total cholesterol was $4.0 \pm 0.9 \mathrm{mmol} / \mathrm{L}$, LDL cholesterol $2.3 \pm 0.7$ $\mathrm{mmol} / \mathrm{L}$ (maximum $4.5 \mathrm{mmol} / \mathrm{L}$ ) and triglycerides $1.0 \pm 0.5$ $\mathrm{mmol} / \mathrm{L}$ (maximum $2.7 \mathrm{mmol} / \mathrm{L}$ ). Nine $(9.4 \%)$ children had an $\mathrm{LDL}$ cholesterol $\geq 3.5 \mathrm{mmol} / \mathrm{L}$, and two $(2.1 \%) \geq 4.0$ $\mathrm{mmol} / \mathrm{L}$. Clinical information was provided on $58(60.4 \%)$ requests; $53(91.4 \%)$ stating 'isotretinoin' or 'acne'. 'Isotretinoin' or 'acne' was mentioned on five (1.4\%) of the remaining 348 lipid profiles not requested by a dermatologist.

Dermatologists already detect some children with homozygous FH, who usually have an LDL cholesterol $>13 \mathrm{mmol} /$ $\mathrm{L}$ and may have coronary, aortic and valvular atherosclerosis and events in their early teenage years, and who present for investigation of cutaneous xanthomata. ${ }^{6}$ The Australasian College of Dermatologists recommend lipid testing prior to and after starting isotretinoin, ${ }^{8}$ as this treatment can lead to elevations of triglyceride and cholesterol in some patients, who are then more likely to have dyslipidaemia and the metabolic syndrome later in life. ${ }^{10}$ Dermatologists order a significant number of lipid profiles in children and adolescents.

The case and data presented suggest that dermatologists could play an important role in detecting FH and help close this gap in preventative cardiology. Childhood is the optimal time to differentiate individuals with FH using LDL cholesterol concentrations, and, if treated, can lead to the greatest reductions in lifetime LDL cholesterol exposure. Therefore, there is potential to significantly increase the detection of $\mathrm{FH}$ in the community through collaboration between the chemical pathologist and dermatologist, especially if combined with testing the parents (reverse cascade screening), ${ }^{5}$ and other first degree family members.

The community laboratory could also assist dermatologists by appending an interpretative comment to the lipid profile of children found to be at risk (LDL cholesterol $\geq 3.5 \mathrm{mmol} / \mathrm{L}$ ) highlighting those individuals at potential risk for $\mathrm{FH}$, which has previously been shown to significantly improve the detection rates. ${ }^{11}$ However, it is likely that an FH awareness and education campaign is required for dermatologists to optimise detection, as significant gaps in FH knowledge have previously been uncovered in primary care, specialists and allied health professionals. ${ }^{2}$

The diagnosis and management of FH will need to be conducted within relevant regional guidelines. ${ }^{1,5,6}$ A diagnostic approach modified from the EAS child and adolescent 
Table 1 Approach to identifying children with FH, modified from European Athersclerosis Society child and adolescent guidelines ${ }^{6}$

\begin{tabular}{ll}
\hline LDL cholesterol & Action required \\
\hline$\geq 5.0 \mathrm{mmol} / \mathrm{L}$ & Repeat lipid profile with TSH, liver and kidney \\
function tests and urine protein to exclude \\
common secondary causes of elevated \\
LDL-c. \\
Refer to regional lipid service if repeat LDL-c \\
Determine if a parent has a history of \\
elevated LDL-c or premature CVD (males \\
$<55$ years, females $<60$ years). \\
Repeat lipid profile with TSH, liver and \\
kidney function tests and urine protein to \\
exclude common secondary causes of \\
elevated LDL-c. \\
Refer to regional lipid service if repeat LDL- \\
c $\geq 4.0$ mmol/L and a parent has elevated \\
LDL-c or premature CVD. \\
Repeat lipid profile with TSH, liver and \\
kidney function tests and urine protein to \\
exclude common secondary causes of \\
elevated LDL-c. \\
Enquire if a parent has been diagnosed with \\
FH. \\
Refer to regional lipid service if repeat LDL- \\
c $\geq 3.5$ mmol/L and a parent has been \\
diagnosed with FH. \\
\hline $3.5-3.9 \mathrm{mmol} / \mathrm{L}$ \\
\end{tabular}

CVD, cardiovascular disease; FH, familial hypercholesterolaemia; LDL-c, low density lipoprotein cholesterol; TSH, thyroid stimulating hormone.

guidelines ${ }^{6}$ is presented in Table 1 . Approximately $10 \%$ of children would require additional questions regarding family history and/or repeat lipid testing, which should not overly burden a practising dermatologist.

In conclusion, dermatologists can play a significant role in the detection of FH by incorporating opportunistic screening for $\mathrm{FH}$ into their guidelines for managing isotretinoin therapy. However, further research is required to explore this potential method of opportunistic FH detection.

Conflicts of interest and sources of funding: The authors state that there are no conflicts of interest to disclose.

\section{Aaron J. Frederiks ${ }^{1}$, John R. Burnett ${ }^{1,2,3}$, Gerald F. Watts ${ }^{1,2}$, Sujith P. Kumarasinghe ${ }^{1}$, Damon A. Bell Bel, $^{1,2,4}$}

${ }^{1}$ School of Medicine, University of Western Australia, Perth, WA, Australia; ${ }^{2}$ Lipid Disorders Clinic, Cardiometabolic Service, Department of Cardiology, Royal Perth Hospital, Perth, WA, Australia; ${ }^{3}$ Department of Clinical Biochemistry, PathWest Laboratory Medicine WA, Royal Perth Hospital and Fiona Stanley Hospital Network, Perth, WA, Australia; ${ }^{4}$ Department of Clinical Biochemistry, Clinipath, Osborne Park, WA, Australia

Contact A/Prof Damon A. Bell.

E-mail: damon.bell@health.wa.gov.au

1. Nordestgaard BG, Chapman MJ, Humphries SE, et al. Familial hypercholesterolaemia is underdiagnosed and undertreated in the general population: guidance for clinicians to prevent coronary heart disease: consensus Statement of the European Atherosclerosis Society. Eur Heart J 2013; 34: 3478-90.
2. Lan NSR, Martin AC, Brett T, et al. Improving the detection of familial hypercholesterolaemia. Pathology 2019; 51: 213-21.

3. Sjouke B, Kusters DM, Kindt I, et al. Homozygous autosomal dominant hypercholesterolaemia in The Netherlands: prevalence, genotypephenotype relationship, and clinical outcome. Eur Heart J 2015; 36: $560-5$.

4. Luirink IK, Wiegman A, Kusters DM, et al. 20-year follow-up of statins in children with familial hypercholesterolaemia. N Engl J Med 2019; 381: $1547-56$.

5. Watts GF, Gidding S, Wierzbicki AS, et al. Integrated guidance on the care of familial hypercholesterolaemia from the International $\mathrm{FH}$ Foundation. Int J Cardiol 2014; 171: 309-25.

6. Wiegman A, Gidding SS, Watts GF et al. Familial hypercholesterolaemia in children and adolescents: gaining decades of life by optimizing detection and treatment. Eur Heart J 2015; 36: 2425-37.

7. Bell DA, Hooper AJ, Bender R, et al. Opportunistic screening for familial hypercholesterolaemia via a community laboratory. Ann Clin Biochem 2012; 49: 534-7.

8. The Australasian College of Dermatologists. The Australasian College of Dermatologists Position Statement: Isotretinoin for Treatment of Acne. Sydney: The Australasian College of Dermatologists; 2018 https://www.dermcoll.edu.au/wp-content/uploads/ACD-PositionStatement-isotretinoin-June-2018.pdf

9. Barth JH, Macdonald-Hull SP, Mark J, et al. Isotretinoin therapy for acne vulgaris: a re-evaluation of the need for measurements of plasma lipids and liver function tests. Br J Dermatol 1993; 129: 704-7.

10. Rodondi N, Darioli R, Ramelet AA, et al. High risk for hyperlipidemia and the metabolic syndrome after an episode of hypertriglyceridemia during 13-cis retinoic acid therapy for acne: a pharmacogenetic study. Ann Intern Med 2002; 136: 582-9.

11. Bender R, Edwards G, McMahon J, et al. Interpretative comments specifically suggesting specialist referral increase the detection of familial hypercholesterolaemia. Pathology 2016; 48: 463-6.

DOI: https://doi.org/10.1016/j.pathol.2020.07.007

\section{Rapid detection of enteric pathogens in a medical assistance team by real-time multiplex PCR}

Sir,

Diarrhoeal diseases acquired through contact with unhygienic conditions and contaminated food pose an ongoing risk to the health of travellers around the world. ${ }^{1}$ A range of pathogens are known to cause diarrhoeal disease in travellers with Escherichia coli pathotypes and Shigella considered some of the most important. ${ }^{1-3}$ 'Traveller's diarrhoea' is usually associated with travel from developed to underdeveloped areas of the world for a period of $<2$ weeks. ${ }^{1}$ While the effects of diarrhoeal disease are typically mild (e.g., tolerable, no impact on activities), moderate (substantial symptoms; impacts on activity) to severe (dysentery; incapacitating) symptoms may develop. ${ }^{1}$ Military and nonmilitary personnel deployed to underdeveloped regions from developed countries face similar problems to the average traveller ${ }^{2}$ with the cumulative time lost having the potential to significantly impact operations. ${ }^{4}$ In deployed settings, accurate and rapid surveillance and early treatment are thus essential to minimise loss of duty days.

In December 2019, an Australian medical assistance team (AUSMAT) was internationally deployed to a South Pacific island nation, to assist local health services in managing an ongoing outbreak of viral illness amongst the population. The 32 person team consisted of civilian doctors, nurses, paramedics and logistics specialists with expertise in a range of skills including infectious diseases and microbiology. Mission diagnostic equipment included a BioFire FilmArray 